\title{
FIXED POINTS AND BEST APPROXIMATIONS FOR MEASURABLE MULTIFUNCTIONS WITH STOCHASTIC DOMAIN
}

\author{
NIKOLAOS S. PAPAGEORGIOU
}

\begin{abstract}
In this paper we prove a best approximation and a random fixed point theorems for Hausdorff continuous multifunctions with stochastic domain. Our result extend several earlier ones existing in the literature. We also show that in Engl [3] some of the hypotheses can be weakened.
\end{abstract}

\section{Introduction}

In this note we prove a fixed point principle and a best approximation theorem analogous to Reich's results [10]. This is done for a class of continuous multifunctions with stochastic domain. Our results are general enough to incorporate earlier works on this subject, like those of Itoh [5], Engl [3], Sehgal-Waters [13], Sehgal-Singh [12], Papageorgiou [9], Lin [8], and Xu [15].

\section{Preliminaries}

Let $(\Omega, \Sigma)$ be a measurable space and $X$ a separable Banach space. We will be using the following notations:

$$
P_{f(c)}(X)=\{A \subseteq X: \text { nonempty, closed, (convex) }\} .
$$

Received June 8, 1991; revised February 25, 1992.

1980 AMS subject classification: $60 \mathrm{H} 25,47 \mathrm{H} 10$

Key words and phrases: Measurable multifunction, stochastic domain, measurable selection, Souslin family, Hausdorff continuity, random fixed point. 
and

$$
P_{k(c)}(X)=\{A \subseteq X: \text { nonempty, compact, (convex) }\}
$$

A multifunction $F: \Omega \rightarrow P_{f}(X)$ is said to be measurable, if for all $x \in X$ the $\mathbb{R}_{+}$-valued function $\omega \rightarrow d(x, F(\omega))=\inf \{\|x-z\|: z \in \mathbb{F}(\omega)\}$ is measurable. Recall that a measurable function $f: \Omega \rightarrow X$ is said to be a "measurable selector" of $F(\cdot)$, if for all $\omega \in \Omega, f(\omega) \in F(\omega)$. It turns out (see Wagner [14], theorem 4.2), that this definition of measurability is equivalswt to saying that there exist messumble selectors $f_{n}: \Omega \rightarrow X r \geq 1$ of $F(\cdot)$, s.t. for all $\omega \in \Omega F(\omega)=$ $\left\{f_{n}(\omega): n=1,2,3, \ldots\right\}$, or that for every $U \subseteq X$ nonempty open $F^{-}(U)=$ $\{\omega \in \Omega: F(\omega) \cap U \neq \emptyset\} \in \Sigma$. Following schäl [11] and Engl [3], we will say that $F: \Omega \rightarrow P_{f}(X)$ is "separable" if it is measurable and there exists a countable set $D \subseteq X$ s.t. for all $\omega \in \Omega, \overline{D \cap F(\omega)}=F(\omega)$.

Let $F: \Omega \rightarrow P_{f}(X)$ be a measurable multifunction and let $G r F=\{(\omega, x) \in$ $\Omega \times X: x \in F(\omega)\}$ (the graph of $F(\cdot)$ ). We know (see Wagner [14], theorem 4.2) that $G r F \in \Sigma \times B(X)$, with $B(X)$ being the Borel $\sigma$-field of $X$. Then $T: G r F \rightarrow 2^{X}\{9\}$ is a measurable multifunction with stochastic domain $F(\cdot)$ if and onty if for all $U \subseteq X$ open, $\{\omega \in \Omega: T(\omega, x) \cap U \neq \emptyset, x \in F(\omega)\} \in \Sigma$. We will say that $I(\cdot, \cdot)$ is an $h$-continuous measurable multifunction with stochastic domain $F(\cdot)$, if in addition for every $\omega \in \Omega$, the multifunction $x \rightarrow T(\omega, x)$ is $h$-continuins on $F(\omega)$ (see definition below).

On $P_{f}(X)$ we an 3 efine a generalized metric, known in the literature as the Hansdorff metric, by setting

$$
h(A, B)=\max \left[\sup _{a \in A} d(a, B), \sup _{b \in B} d(b, A)\right]
$$

where $d(a, B)=\inf \{\|a-b\|: b \in B\}$ and $d(b, A)=\inf \{\|b-a\|: a \in A\}$. It is well known that $\left(P_{f}(X), h\right)$ is a complete metric space and $\left(P_{k c}(X), h\right)$ a closed (hence complete) subset of it. In fact, $P_{k c}(X), h$ ) is also separable, while $\left(P_{f}(X), h\right)$ is not. If $Y$ is a topological space, a multifunction $F: Y \rightarrow P_{f}(X)$ is said to be Hausdorff continuous ( $h$-continuous) if and only if it is continuous as a map from $Y$ into the metric space $\left(P_{f}(X), h\right)$. 
Let $Z$ be a set and denote by $P(Z)$ s a collection of subsets of $Z$. Denote by $\hat{i}$ the set of all infinite sequences of positive integers and by $\hat{N}_{0}$, the set of all finite sequences of positive integers. A set $A \subseteq Z$ is said to be obtained from $P(Z)$ by applying the Souslin operation, if there exists a map $k: \hat{N}_{0} \rightarrow$ $P(Z)$ s.t. $A=\bigcup_{r \in \hat{N}} \bigcap_{n=1}^{\infty} k(r \mid n)$, where $r \mid n$ denotes the first $n$-elements of $r \in \hat{N}$ (see Jacobs [6]). Note that the union in the Souslin speration is uncountable. So if $P(Z)$ is a $\sigma$-field, then $A$ may be outside of $P(Z)$. If however $P(Z)$ is closed under the Souslin operation, then we say that $P(Z)$ is a Souslin family. For example every complete $\sigma$-field is a Souslin family (see Wagner [14]).

Let $(\Omega, \Sigma)$ be a measurable space, $Y$ a separable metrizable space and $Z$ a metrizable space. A function $f: \Omega \times Y \rightarrow Z$ is said to be a Caratheodory function, if for all $y \in Y, \omega \rightarrow f(\omega, y)$ is measurable and for all $\omega \in \Omega, y \rightarrow f(\omega, y)$ is $1:$ is well-known that such a function is jointly measurable; i.e. $(\omega, y) \rightarrow f(\omega, y)$ is $(\mathrm{E} \times B(Y), B(Z))$-measurable.

\section{Main Results}

We start with an approximation result, which can be viewed as the stochastic version of a result originally obtained by Reich [10]. Our result also generalizes theorem 2 of Sehgal-Singh [12], where the multifunction had a deterministic domain.

Wheorem 3.1. If $(\Omega, \Sigma)$ is a measurable space with $\Sigma$ a Souslin family, $X$ is a separable Banach space, $K: \Omega \rightarrow P_{k c}(X)$ is a separable multifunction and $T: G r K \rightarrow P_{k c}(X)$ is an h-continuous, measurable multifunction with stochastic domain $K(\cdot)$, then there exists a measurable map $x: \Omega \rightarrow X$ s.t. for all $\omega \in \Omega$ $x(\omega) \in K(\omega)$ and $d(x(\omega), T(\omega, x(\omega)))=\delta(K(\omega), T(\omega, x(\omega)))=\inf \{\|v-w\|: v \epsilon$ $K(\omega), w \in T(\omega, x(\omega))\}$.

Proof. Using corollary 3.1 of Kandilakis-Papageorgiou [7], we can find a multifunction $\hat{T}: \Omega \times X \rightarrow P_{k c}(X)$ s.t. $\omega \rightarrow \hat{T}(\omega, x)$ is measurable, $x \rightarrow \hat{T}(\omega, x)$ 
is $h$-continuous and $\left.\hat{T}\right|_{G r K}=T$. Then consider the multifunction $H: \Omega \rightarrow 2^{X}$ defined by

$$
H(\omega)=\{y \in K(\omega): d(y, \hat{T}(\omega, y))=\delta(K(\omega), \hat{T}(\omega, y))\} .
$$

From lemma 1.6 of Reich [10], we know that for every $\omega \in \Omega, H(\omega) \neq \emptyset$.

Let $\varphi: \Omega \times X \rightarrow \mathbb{R}_{+}$be defined by $\varphi(\omega, x)=d(x, \hat{T}(\omega, x))$. Clearly $\varphi(\cdot, \cdot)$ is a Caratheodory function. Also let $k_{n}: \Omega \rightarrow X n \geq 1$ be measurable selectors of $K(\cdot)$ s.t. for all $\omega \in \Omega, K(\omega)=\overline{\left\{k_{n}(\omega): n=1,2,3, \ldots\right\}}$ (see section 2). Then we have:

$$
\delta(K(\omega) \hat{T}(\omega, y))=\inf _{n \geq 1} \inf _{z \in \hat{T}(\omega, y)}\left\|k_{n}(\omega)-z\right\| .
$$

Set $\psi_{n}(\omega, y)=\inf _{z \in \hat{T}(\omega, y)}\left\|k_{n}(\omega)-z\right\|$. Theorem 6.1 of [7] tells us that for every $n \geq 1, \omega \rightarrow \psi_{n}(\omega, y)$ is measurable, while proposition 23, p. 120 of Aubin-Ekeland [1] tells us that for every $n \geq 1, y \rightarrow \psi_{n}(\omega, y)$ is continuous. So $\psi_{n}(\cdot, \cdot) n \geq 1$ is a Caratheodory function, hence jointly measurable. Thus $\psi(\omega, y)=\inf _{n \geq 1} \psi_{n}(\omega, y)$ is a measurable function s.t. for all $\omega \in \Omega, \psi(\omega, \cdot)$ is upper semicontinuous (see for example Bertsekas-Shreve [2], lemma 7.14, p. 147). Let $\eta(\omega, y)=\varphi(\omega, y)-$ $\psi(\omega, y)$. Then clearly $\eta(\cdot, \cdot)$ is jointly measurable, and for every $\omega \in \Omega, \eta(\omega, \cdot)$ is lower semicontinuous. Also note that for all $(\omega, y) \in \Omega \times X, \eta(\omega, y) \geq 0$. Observe that $H(\omega)=\{y \in K(\omega): \eta(\omega, y) \leq 0\}$. Hence for all $\omega \in \Omega, H(\omega) \in P_{k}(X)$.

Set $L_{0}(\omega)=\{y \in X: \eta(\omega, y) \leq 0\}=\{y \in X: \eta(\omega, y)=0\}$ and observe that

$$
H(\omega)=K(\omega) \cap L_{0}(\omega) .
$$

Since $\eta(\cdot, \cdot)$ is jointly measurable, we have $G r L_{0}=\{(\omega, y) \in \Omega \times X$ : $\eta(\omega, y)=0\} \in \Sigma \times B(X)$. Since by hypothesis $\Sigma$ is Souslin family, from theorem $4.2(g)$ of Wagner [14], we get that $L_{0}(\cdot)$ is measurable $\Rightarrow H(\cdot)$ is measurable. Applying the Kuratowski-Ryll Nardzewski selection theorem (see Wagner [14]), we get $x: \Omega \rightarrow X$ measurable s.t. for all $\omega \in \Omega, x(\omega) \in H(\omega)$. Then $x(\omega) \in K(\omega)$ and $d(x(\omega), T(\omega, x(\omega)))=\delta(K(\omega), T(\omega, x(\omega)))$ for all $\omega \in \Omega$. $\quad$ Q.E.D.

The next result is a general fixed point principle, that incorporates all random fixed point theorems involving continuous multifunctions. In particular, it 
contains as special cases the fixed point theorem of Itoh [5], theorems 8, 13, 14 and 15 of Engl [3], theorem 2 of Sehgal-Waters [13], corollary 1 of Sehgal-Singh [12], theorem 6 of Papageorgiou [9], theorems 4 and 5 of Lin [8] and theorems 1 , 2,3 and 4 of Xu [15]. From the above works, only Engl [3], considered multifunctions with stochastic domain. However he assumed that there exists a $\sigma$-finite measure $\mu(\cdot)$ on $(\Omega, \Sigma)$, that $\operatorname{int} K(\omega) \neq \emptyset \mu$-a.e. and that the multifunction $\omega \rightarrow \operatorname{int} K(\omega)$ admits a measurable selector $x_{0}(\cdot)$. Furthermore, his random fixed point satisfies $x(\omega) \in T(\omega, x(\omega)) \mu$-a.e. and not for all $\omega \in \Omega$. Our result drops all the above extra hypotheses of Engl [3] and obtains a random fixed point for every $\omega \in \Omega$ by assuming that $\Sigma$ is Souslin family. In addition, our proof is considerably simpler and shorter than that of Engl [3]. Finally in proposition 3.3 , we show that the selector hypothesis on the multifunction $\omega \rightarrow \operatorname{int} K(\omega)$ is superfluous, since it is automatically implied by the other hypotheses that Engl [3] made.

Theorem 3.2. If $(\Omega, \Sigma)$ is a measurable space with $\Sigma$ a Souslin family, $X$ a separable Banach space, $K: \Omega \rightarrow P_{f}(X)$ a separable multifunction, $T: G r K \rightarrow$ $P_{k c}(X)$ is an h-continuous measurable multifunction with stochastic domain $K(\cdot)$ and for every $\omega \in \Omega H(\omega)=\{x \in K(\omega): x \in T(\omega, x)\} \neq \emptyset$, then there exists a measurable map $x: \Omega \rightarrow X$ s.t. for all $\omega \in \Omega x(\omega) \in K(\omega)$ and $x(\omega) \in T(\omega, x(\omega))$.

Remark. So this result says that under the above hypotheses, "deterministic" solvability of the fixed point problem implies "stochastic" solvability of it.

Proof. Applying corollary 1.3 of [7], we get $\hat{T}: \Omega \times X \rightarrow P_{k c}(X)$ a multifunction s.t. $\omega \rightarrow \hat{T}(\omega, x)$ is measurable, $x \rightarrow \hat{T}(\omega, x)$ is $h$-continuous and $\left.\hat{T}\right|_{\text {GrK }}=T$. Let $H: \Omega \rightarrow 2^{X}$ be defined by

$$
H(\omega)=\{x \in K(\omega): x \in \hat{T}(\omega, x)\}
$$

By hypothesis, for all $\omega \in \Omega H(\omega) \neq \emptyset$ and it is easy to check using the $h$-continuity of $\hat{T}(\omega, \cdot)$, that for all $\omega \in \Omega H(\omega) \in P_{f}(X)$. Let $\varphi: \Omega \times X \rightarrow \mathbb{R}_{+}$ 
be defined by $\varphi(\omega, x)=d(x, \hat{T}(\omega, x))$. Clearly $\varphi(\cdot, \cdot)$ is a Caratheodory function. Then note that

$$
G r H=G r K \cap\{(\omega, x) \in \Omega \times X: \varphi(\omega, x)=0\} \in \Sigma \times B(X)
$$

Since $\Sigma$ is a Souslin family, theorem 4.2 of Wagner [14], tells us that $H(\cdot)$ is measurable. So applying the Kuratowski-Ryll Nardzewski selection theorem, we get $x: \Omega \rightarrow X$ measurable s.t. for all $\omega \in \Omega, x(\omega) \in H(\omega)$. Clearly $x(\cdot)$ is the desired random fixed poirt for $T(\cdot, \cdot)$.

Q.E.D.

Finally in the next proposition, we show that in theorem 8 of Engl [3], the hypothesis that there exists a measurable function $x_{0}: \Omega \rightarrow X$ s.t. $x_{0}(\omega) \in$ int $K(\omega) \mu-a . e$. is superfuous.

Proposition 3.3. If $(\Omega, \Sigma, \mu)$ is a $\sigma$-finite measure space, $X$ is a separaite punach space ard $K: \Omega-p_{f}(X)$ is a multifunction s.t. $G r K \in \Sigma \times B(X)$ and int $K(\omega) \neq \emptyset \mu$-a.e., then there exist $x_{0}: \Omega \rightarrow X$ measurable functin s.t. $a_{0}(\omega) \in \operatorname{int} K(\omega) \mu-a . e$.

Proof. Observe that

$$
G \operatorname{Gint} K(\cdot)=\{(\omega, x) \in \Omega \times X: d(x, \operatorname{bdK}(\omega))>0\} \cap \operatorname{Gr} K
$$

where hdK $(\omega)$ denotes the boundary of $K(\omega)$. Note that bdF( $\omega)$ may be empty. In that case as usual $d(x, b d K(\omega))=+\infty$. But from theorem 4.6 of Himmelberg [4], we know that if $D=\{\omega \in \Omega: b d f(\omega)+h\}$ then $D \in 2$ and $\omega-b J(\omega)$ is measurable on $D$. Hence $(\omega, w) \in g \times x: d(x, b / K(\omega))>0\} \cap O r K=$ $\left[\{(\omega, x) \subseteq D \times X: d(x, b d K(\omega))>0\} \cup\left(D^{\circ} \times X\right)\right] \cap G r k \in \Sigma \times B(X)$. (Note that, $(\omega, x) \rightarrow d(x, F(\omega))$ is a Caratheodory function on $D \times X$, hence jointly measurable). Therefore Grint $K \in \Sigma \times B(X)$. Apply Aumann's selection theorem (see Wagner [14], to get $x_{0}: \Omega \rightarrow X$ measurable s.t. $x_{0}(\omega) \in$ $\operatorname{int} K(\omega) \mu-$ a.e.

Q.E.D.

Remark. Note that our hypothesis on $K(\cdot)$ is weaker than that of Engl [3] (theorem 8), since we only assume graph measurability of $K(\cdot)$ and not measur- 
abilty of it. Recall that for a $P_{f}(X)$-valued multifunction measurability implies graph ineasurability.

\section{Acknowledgement}

The anthor wishes to thank the referee for his (her) helpful corrections and remarks.

\section{References}

[1] J.-P. Aubin and I. Ekeland, "Applied Nonlinear Analysis", Wiley, New York (1984).

[2] D. Bertsckas and S. Shreve, "Stochastic Optimal Control: The Discrete Time Case", Ácadernic Press, New York (1978).

[3] H. Engl, "A general stochastic fixed point theorem for continuous random operators on stochastic domains", J. Math. Ancl. Appl. 66, (1978), pp. 220-231.

[4] C. Himmelberg, "Measurable relations", Fundamenta Math. 87, (1971), pp. 59-71.

[5] S. Foh, "A randorn fixed point theorem for a maltivalued contraction mapping", Facific I. Mcth. $38,(1977)$, pp $85-90$.

56 K. Jacobs, "Measure and Integral", Academic Press, New York (1978).

[?] D. Kardiakis and N.S. Papageorgious, "Properties of measurable multifunctions with stochastic domain and their applications", Math. Japonica 35, (1990), pp. 629-643.

[9] T.C. Iin, "Random approximations and random fixed point theorems for non-self maps", Froc. Amer. Math. Soc. 103, (1988), pp. 1129-1135.

(9) A. Papageorgiou, "Pandom fixed point theorems for measurable multifunctions in BaIath spaces", Proc. Amer. Math. Soc. 87 , (1986), pp. 507-514.

[0] S. Feich, "Fixed points in locally convex spaces", Math. Z. 125, (1972), pp. 17.3.

1] M Schat, "A seiection theorem for optimization problems", Arch, Math. 25, (1374), pp. $210 \cdot 224$

[12] V. Selgal and S. Singh, "On random approxinations and a random fixed point theorems for set valued mappings", Proc. Amor. Moth. Soc. 55, 1906? pp. 9\%-94,

[19] $Y$ Sehys and C. Westers, "bone rardom fixed point thoorems for condensing operators", Fron. Amer Math. Soc 90, (1984), pp. 425-429.

[14] D. Wagner, "Survey of measurable selection theorems", SIAM I Contrik OMtion. IS, (1977), pp. 859-903.

[15] H.-K. Xu, "Some random fixed point theorems for condensing and nonexpansive operatrors", Proc. Amer. Math. Soc. 110, (1990), pp. 395-400.

Florida Institute of Technology, Department of Applied Mathematics, 150 W. University Blvd., Melbourne, Florida. 32901-6988, U.S.A. 